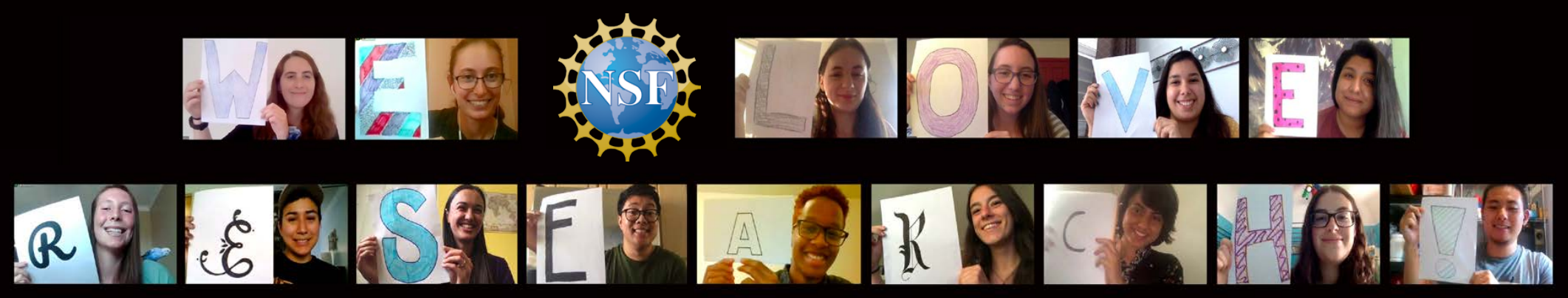

\title{
STRATEGIES FOR RUNNING A SUCCESSFUL VIRTUAL CAREER PANEL
}

\author{
By Janine Barr, Christine Bean, and Janice McDonnell
}

ABOVE. Screenshots of students participating in the Rutgers virtual REU experience.

As part of an eight-week virtual Research Experiences for Undergraduates (REU) program, oceanographers and outreach specialists at Rutgers, the State University of New Jersey, conducted a Virtual Marine Science Career Panel (Ocean Data Labs, 2020). Instead of canceling summer REU programs across the United States (NSF, 2020b), the National Science Foundation (NSF) supported and encouraged academic leadership to develop virtual programming in keeping with each institution's organizational policies concerning the pandemic (NSF, 2020a). Here, we highlight facilitation strategies for running a successful virtual career panel as well as lessons learned that may serve as inspiration for others working to create similar virtual experiences in their own REU programs and beyond.

\section{FACILITATION STRATEGY:}

\section{USING ONLINE TOOLS AND}

\section{BUILDING MENTOR-MENTEE}

\section{RELATIONSHIPS VIRTUALLY}

The Rutgers team invited six marine science professionals from around the United States to participate in the Virtual Marine Science Career Panel. They represented careers from the education and outreach, federal government, industry, nonprofit, research, and state government sectors. To maximize the time students had to interact with each panelist in the limited virtual environment, the onehour panel was structured differently than a traditional career panel where panelists summarize their work trajectories and then respond to questions. Here, the Rutgers team adopted a structure that matched small groups of students with panelists to help make the virtual environment as conversational as possible and foster meaningful connections between students and panelists. This structure was implemented using the video breakout room feature in Zoom Meetings available through a paid Pro account.

To match students with panelists, the Rutgers team asked each panelist to complete a questionnaire prior to the virtual event (see online supplemental materials). The seven questions presented were geared toward understanding the panelists' career paths and how their backgrounds in marine science brought them to their current positions. Students were provided the completed questionnaires a couple days in advance of the panel and were asked to provide feedback to the Rutgers team on their priorities regarding whom they wished to speak with in the small breakout sessions. Students submitted their rankings through a short survey created for free using Google Forms.

The panel itself began with the panelists providing brief overviews of their career disciplines. The panelists were then placed in breakout rooms where they each interacted with three different groups of two to three students for periods of 13 minutes. With 15 participants, each student got to interact with three panelists (Figure 1). Once the three breakout sessions had concluded, all the panelists and students returned to the main Zoom room for concluding remarks. For additional details regarding the logistics of the panel, please contact the authors.

\section{KEY INGREDIENTS FOR THE VIRTUAL MARINE SCIENCE CAREER PANEL}

The Rutgers team has identified three key ingredients that helped make this Virtual Marine Science Career Panel a success.

1. Recruiting panelists from a wide geographic range. An advantage of running a virtual career panel was that the Rutgers team was able to invite professionals who work in geographically distant locations (e.g., Houston, Texas, and Washington, DC) and 
who represent a more diverse range of career paths and experiences than would be possible for a one-hour in-person panel.

2. Engaging panelists and students before the panel using a standardized questionnaire. Instead of preparing slides or formal introductory remarks, each panelist was asked to fill out a standardized questionnaire nine days prior to the panel (see online supplementary materials). The questionnaires were shared with the students two days prior to the panel, allowing them to make informed choices about which panelist they wanted to speak with, and there was more time to engage with each panelist because introductory remarks were not extensive.

3. Setting up smaller conversations using breakout rooms. Panelists and students provided positive feedback regarding the structure of the virtual career panel's breakout rooms. Each 13-minute breakout session yielded unique conversations focused on that smaller group of students' interests. This structure also offered an opportunity for students to build their pro- fessional networks. It provided each student with a personal connection to a panelist, helping the student see that panelist's career path as more attainable.

\section{LESSONS LEARNED}

While the Virtual Marine Science Career Panel was largely a success at Rutgers, we offer several additional considerations that would improve similar career panels conducted at other institutions.

If the virtual career panel is one component of a longer online program, institutions may benefit from creating a list of "norms" with their students to help them feel more comfortable in a new virtual space and to provide a sense of community (KONU, 2020). Norms here refer to online etiquette that students and their instructors can brainstorm and agree to follow together (e.g., deciding if everyone should always have their video on, or if calling in is okay, and committing to ending each session on time). It may be helpful to designate funds for purchasing Wi-Fi hotspots to distribute to students who do not have access to high-speed Internet. The availability of hotspots

\begin{tabular}{|l|l|l|l|l|l|}
\hline Timeslot & $4: 00-4: 10 \mathrm{pm}$ & $4: 10-4: 23 \mathrm{pm}$ & $4: 23-4: 36 \mathrm{pm}$ & $4: 36-4: 49 \mathrm{pm}$ & $4: 49-5: 00 \mathrm{pm}$ \\
\hline Main Zoom Room & & & & & \\
& & & & & \\
\hline
\end{tabular}

FIGURE 1. Locations of the Rutgers team (gray boxes, $n=3$ ), panelists (orange boxes, $n=6$ ), and students (blue boxes, $n=15$ ) as they participated in the Virtual Marine Science Career Panel. Each box represents a unique individual. The panel began at four in the afternoon with 10 minutes of introductory remarks, followed by three 13-minute breakout sessions, and 11 minutes for concluding remarks. Panelists remained in their breakout rooms for the three breakout sessions, and the students were moved between rooms. Two Rutgers team members remained in the main Zoom meeting room to coordinate moving students to their assigned breakout rooms while the third team member moved between breakout rooms to ensure there were no technical difficulties or other issues. facilitates the students' use of video conferencing and helps them fully participate in the virtual experience. Finally, consider holding a virtual career panel for at least one hour and 15 minutes when following the structure discussed above. A slightly longer time devoted to a career panel would ensure sufficient time to move students between breakout rooms without compromising their time with each panelist.

Although it was only a small part of the eight-week summer REU program at Rutgers, the successful implementation of our virtual career panel can be used as a template for other career panels in the ocean sciences, whether associated with REUs, large conferences, university alumni programs, or scientific societies. @

\section{ONLINE SUPPLEMENTARY MATERIALS}

The supplementary materials are available online at https://doi.org/10.5670/oceanog.2020.220.

\section{REFERENCES}

KONU. 2020. Enabling Humanity on Zoom-How to Facilitate Deep Learning Virtually. https://konu.org/ blog-en/2020/3/16/enabling-humanity-on-zoomhow-to-faciliate-deep-learning-virtually.

NSF (National Science Foundation). 2020a.

Frequently Asked Questions (FAQs) About the Coronavirus Disease 2019 (COVID-19) for Research Experiences for Undergraduates (REU) Sites, Research Experiences for Teachers (RET) Sites, International Research Experiences for Students (IRES) Sites, and Similar Activities. https://www.nsf. gov/bfa/dias/policy/covid19/covid19faqs_reu.pdf. NSF. 2020b. Research Experiences for Undergraduates (REU), https://www.nsf.gov/funding/ pgm_summ.jsp?pims_id=5517.

Ocean Data Labs. 2020. Data Labs 2020 Virtual Research Experience for Undergraduates (REU), https://datalab.marine.rutgers.edu/2020virtual-reu/.

\section{ACKNOWLEDGMENTS}

The authors would like to thank the students and mentors for their participation, Lisa Auermuller, and their fellow Rutgers team members for making this program a reality: Josh Kohut, Sage Lichtenwalner, Daphne Munroe, and Anthony Vastano. This project was funded by National Science Foundation Grant No. OCE-1831625.

\section{AUTHORS}

Janine Barr (jmb883@hsrl.rutgers.edu) is a graduate student pursuing a master of science degree in oceanography at Rutgers University and is a trainee in the Rutgers Coastal Climate Risk and Resilience Program. Christine Bean (bean@marine.rutgers.edu) is STEM Program Administrator at Rutgers University. Janice McDonnell (mcdonnel@marine.rutgers.edu) is a STEM agent in the Department of Youth Development, and Associate Professor, Rutgers Cooperative Extension, Rutgers University. 